
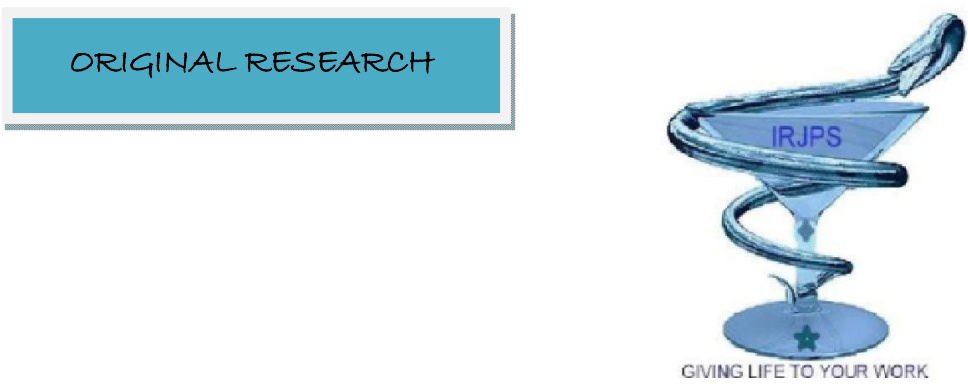

\title{
IMPACT OF PATIENT COUNSELLING BY CLINICAL PHARMACIST IN IMPROVING KAP ( KNOWLEDGE, ATTITUDE AND PRACTISE ) OF CAREGIVERS OF PAEDIATRIC POPULATION REGARDING ANTIBIOTIC USE FOR RESPIRATORY TRACT INFECTION
}

Fiona Markose, Junymon Chacko, Nidhi. S.S.Thampy

Sree Krishna College of pharmacy, Parassala, Thiruvananthapuram, India

\begin{abstract}
:
Background: Upper respiratory tract infections .(URTIs)are common in children. Patients' knowledge and expectations may influence the prescription of antibiotics. Therefore, providing evidence-based information on cause of symptoms, self-management and treatment is essential. Antibiotic abuse in respiratory tract infection is an important factor contributing to development of antibiotic resistance and therefore judicious use of antibiotics in children is crucial However, providing information during consultations is challenging. Patient counselling enables to optimise the antibiotic use and minimize antibiotic resistance.
\end{abstract}

Methodology: On the basis of the study, a total of 200 paediatric patients prescribed with antibiotics, who met the inclusion and exclusion criteria of our study were randomly selected and their outpatient and inpatient record were monitored and recorded for a period of 6 months. The data was then analyzed.

Results:The knowledge of parents of paediatric population increased from mean score of $2.9 \pm 1.2$ SD ( before counselling ) to $6.4 \pm 0.5$ ( after counselling) in the test group and in control there was only a slight increase. Similarly the attitude and practise of patients in case had a increase after counselling and in control there was only a slight increase.

Conclusion: Clinical pharmacists need to investigate more time in educating mostly physicians on the potential benefit of reducing antibiotic prescription for childrens with URTI.The use of information leaflets in general practice consultations are effective in reducing antibiotic prescription by GPs, and actual antibiotic use by patients and their intention to reconsult for future similar episodes of illness.

Key Words: Clinical pharmacist, Respiratory tract infection, KAP, Patient counselling.

Corresponding Author: Fiona Markose

Email: fionamarkose2@gmail.com
Indian Research Journal of Pharmacy and Science; 11(2016) 857-860; Journal Home Page: https://www.irjps.in 


\section{INTRODUCTION:}

According to WHO NICE guideline, Respiratory tract infection (RTI) is defined as any infectious disease of the upper or lower respiratory tract. Upper respiratory tract infections include the common cold, pharyngitis or tonsillitis, acute rhinitis etc. Lower RTIs include acute bronchitis, bronchiolitis, pneumonia etc. These conditions are largely selflimiting and complications are likely to be rare if antibiotics are withheld. ${ }^{(1)}$

Respiratory tract infections are particularly common in children and are major cause of morbidity. It has high cost to society, being responsible for absenteeism from school and work and unnecessary medical care and is occasionally associated with serious sequelae. Inappropriate use of antimicrobial agents for viral respiratory tract infection (most URTIs) is common, leading to constant increase of bacterial respiratory pathogens. ${ }^{(2)}$

\section{ANTIBIOTICS IN RESPIRATORY TRACT INFECTIONS}

Antibiotics are commonly prescribed for RTIs in children. They are key drugs for treatment of infections and are among the most commonly prescribed drugs in the paediatric department.

\section{KAP AND PATIENT COUNSELLING}

KAP is a quantitative method based on predetermined questions formatted in standardized questionnaires which is used to determine the extent of a known situation, confirm or disprove a hypothesis and provide new tangents of a situations reality.

Patient counselling:This may help to reduce the pressure on physicians to prescribe antibiotics by the parents, reducing the spread of resistant bacterial pathogens through judicious antimicrobial use ,reducingantibiotic overuse or misuse.

Rational use of antibiotics: requires that patients receive medications appropriately to their clinical needs, in doses that meet their own individual requirements for an adequate period of time ,at the lowest cost to them and their community.

\section{MATERIALS AND METHODS:}

This prospective observational study was conducted in paediatric department of a teritiary care hospital after obtaining approval from the Institutional ethics committee. The study included both inpatients and outpatients of both gender with an age $\leq 12$ yrs. The main restriction to study was one with incomplete medical records and those who are unwilling to participate in the study. The study also excluded paediatric patients with no follow up.

Based on inclusion and exclusion criteria 200 prescriptions issued during a period of 6months were randomly collected and relevant data was analyzed and tabulated in a specifically designed data collection form and sideeffectchecklist. Patients demographic details, laboratory and clinical information were collected during outpatient and inpatient hours and by reviewing medical records. All the data collected were coded as per variables and entered in SPSS data sheet.

\section{RESULT:}

The knowledge of patients increased from mean score of $2.9 \pm 1.2 \mathrm{SD}$ before counselling to $6.4 \pm 0.5$ SD after counselling in the test / case group and in the control group, there was only a slight increase (by a score of $0.3 \pm 0.2 \mathrm{SD}$ only).

\section{Graph 1: Comparison of knowledge based on group}

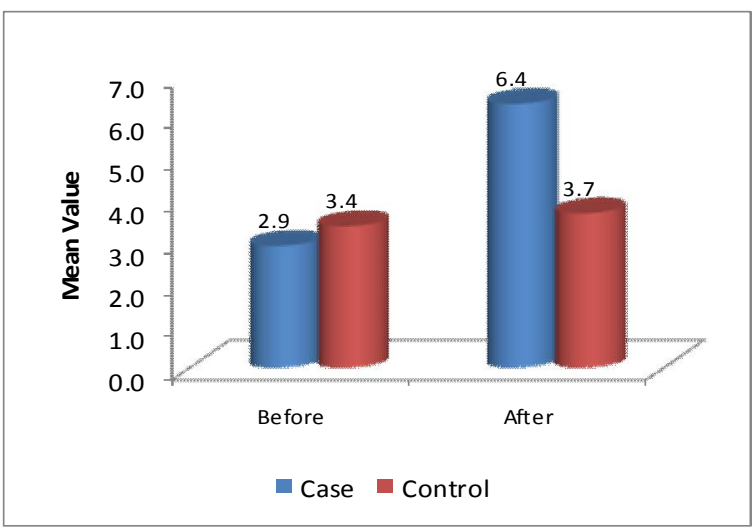

Attitude mean score before counseling in the test or case group was found to be $21 \pm 3.6 \mathrm{SD}$ and after counseling was $33.3 \pm 1.4 \mathrm{SD}$ and in control group, there was an increase from $21.4 \pm 2.5 \mathrm{SD}$ to $22.7 \pm 2.3$ 
SD, which was relatively non-significant as compared to increase of score in cases.

\section{Graph 2: Comparison of attitude based on group}

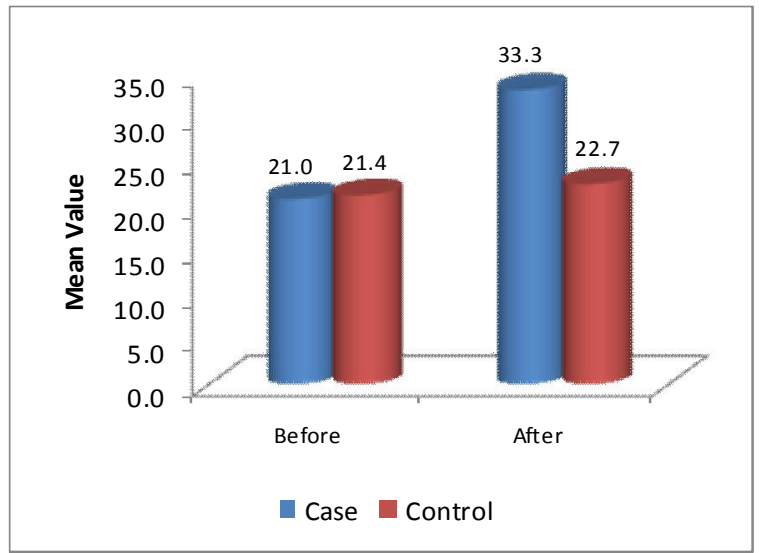

Practice concerns the ways that the parents demonstrate their knowledge and attitude through their actions. In test population, there was an increase in mean value of practice score from $40.5 \pm 4.1 \mathrm{SD}$ before counseling to $53.5 \pm 1.3 \mathrm{SD}$ after counseling. In control group, there was only a slight increase,i.e., $42.7 \pm 2.6 \mathrm{SD}$ to $44.2 \pm 2.2 \mathrm{SD}$.

\section{Graph 3: Comparison of practice based on group}

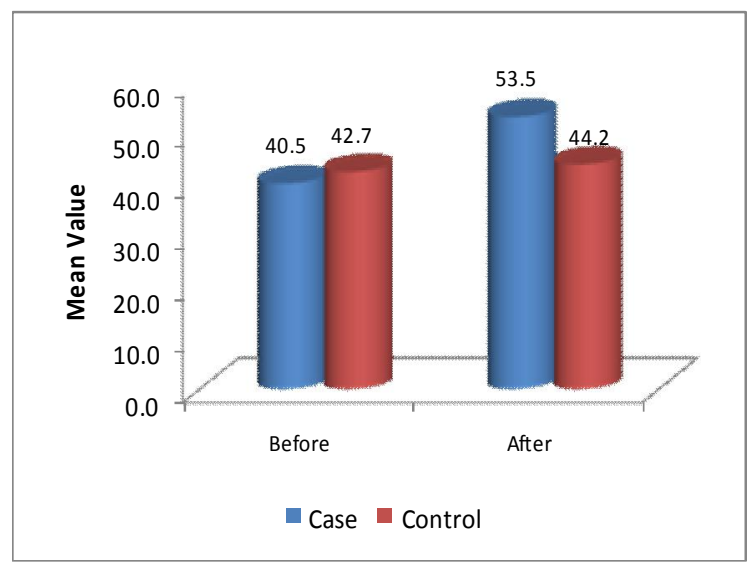

\section{DISCUSSION:}

Most parent's expectation for antibiotic prescription is high, therefore further parental education is needed. Clearly demographic factors such as residence area, ethnicity, parental age, sex, and educational level are associated with knowledge, attitude \& practice concerning judicious use of
antibiotics.Older parental age and higher educational level ware clearly associated with better knowledge. while younger parents are worried about fever, older parents were more likely to worry by altered behaviour of their child during a URTI. ${ }^{(7)}$ In our study most of the parents /caregivers of paediatric population were graduates and had a lower response due to the higher education and most of the caregivers or parents were employed(90\%). Persons more frequently caring for child was grandmother or grandfather or mother. In a similar descriptive study on parents "knowledge, attitude and practice" on antibiotic use and misuse in children with upper respiratory infection, showed that the response rate was lower in areas with higher education. ${ }^{(7)}$ Another similar study conducted by Maria Luisa et.al, on why paediatricians prescribe antibiotics, results of an Italian project, also showed that socioeconomic status such as having a high house hold income or being a doctor or pharmacist can influence the antibiotic prescribing (because these subgroups may be informed about appropriate antibiotic therapy or having greater flexibility in taking time off work to bring their children back to a physician if symptoms do not resolve) in more than $80 \%$ of cases. ${ }^{(2)}$

The two step knowledge, attitude, practice, survey was conducted on most common respiratory tract infections. The first phase of the study aimed to explore knowledge, attitude and practice towards management of RTIs, antibiotic use and antimicrobial resistance. The second phase of study aimed to explore the impact of patient counselling on public.A total of 200 questionnaires were completed. There was an overall increase in average score of KAP in second phase after patient counseling given in the first session. The parents / caregivers of paediatric population had a trusted relationship with the the physician because they were happy with the information provided to them and would not change the paediatrician if antibiotics were used too much or too little. A similar study was conducted by SotiriaPanagakou regarding antibiotic use for URTI in paediatric population.(11) In descriptive study conducted by Andreas regarding knowledge, attitude , and practice on antibiotic use and misuse in children , it was observed that methodology using selfanswered questionnaires increases the precisionof information obtained. ${ }^{(9)}$ 


\section{CONCLUSION:}

Clinical pharmacists need to investigate more time in educating mostly physicians on the potential benefit of reducing antibiotic prescription for childrens with URTI. Clinical pharmacist interventions had a great importance in improving the knowledge, attitude and practice of caregivers/ parents of paediatric

\section{REFERENCE:}

1. https://www.nice.org.uk.

2. Cals JW, Public beliefs on antibiotics and respiratory tract infections:an internet based questionnaire study.Br J Gen Pract 2007,942-947

3. Trepka MJ et.al;The effect of a community intervention trial on parental knowledge and awareness of antibiotic resistence and appropriate antibiotic use in children .Paediatrics 2001,107-109

4. National Institute For Health and Clinical Excellence:Prescribing of antibiotics for self limited respiratory tract infections in children in primary care:Clinical guidance 2008

5. Sonal M, SujaA ,Revikumar KG , Emerging trends in practice of patient counselling-Indian scenario. Indian $\mathrm{J}$ Pharm Practice 2008;1:6-13. population and reducing the emergence of resistance and side effects through proper selection and administration of antibiotics.

\section{ACKNOWLEDGEMENT:}

We would like to thank one and all who directly and indirectly supported us and thus our study was a success.

6. Sotiria G Panagakou, antibiotic use for URTIs in children: A cross sectional survey of knowledge, attitude, practice of parents, BMC Paediatrics 2011, page no: $1471-2431$

7. Andreas Rousounidis, Descriptive study on parent's knowledge, attitudes and practices on antibiotic use and misuse in children with upper respiratory tract infection in Cyprus, IntJ.Environ .public health 2011, page no: 3246-3262.

8. Maria Luisa Moro et, Why do paediatricians prescribe antibiotics? Results of an Italian regional project, BMC Paediatrics(2009), page no: 14712431.

9. Stevenson F, A Systematic Review of Communication between Patients and Health Providers about Medicine-taking and Prescribing. GKT Concordance Unit, Kings College, 2003. 